\title{
Universal Sequence of the Ground States and Energy Level Ordering in Frustrated Antiferromagnetic Rings with a Single Bond Defect
}

\author{
M. AntKowiak*, W. Florek and G. Kamieniarz \\ Adam Mickiewicz University in Poznań, Faculty of Physics, Umultowska 85, 61-614 Poznań, Poland
}

\begin{abstract}
The universal sequence of the ground states for antiferromagnetic frustrated rings with the odd number of the local spins $s$ and a single bond defect $\alpha$ described by the isotropic Heisenberg Hamiltonian is discussed. The LiebMattis energy level ordering in a pentanuclear ring is revealed and the arising magnetisation steps are demonstrated.

DOI: 10.12693 /APhysPolA.131.890

PACS/topics: 75.10.Jm, 75.10.Hk, 75.50.Xx, 03.65.Aa
\end{abstract}

\section{Introduction}

Recently, magnetic molecules have been one of important subjects of investigations due to their potential applications and some fundamental problems of quantum spin systems theory $[1-5]$.

In this paper we concentrate on regular rings with an odd number of identical spins $s_{j}=s, 1 \leq j \leq n$, with one "defect bond", i.e. the quantum spin systems described by the isotropic Heisenberg Hamiltonian

$$
H=\sum_{j=1}^{n-1} \boldsymbol{s}_{j} \cdot \boldsymbol{s}_{j+1}+\alpha \boldsymbol{s}_{n} \cdot \boldsymbol{s}_{1},
$$

where a real number $\alpha$ gives a relative defect magnitude. This model Hamiltonian is inspired by research related to a wide class of chromium-based rings [2-7]. It should be stressed that for $\alpha>0$ such systems are geometrically frustrated $[3,4,8,9]$ and do not satisfy assumptions of the Lieb-Mattis theorem (LMT) for $n>3[4,8,9]$. We note that Kahn [11] restricted the notion of frustration in molecular nanomagnets to the particular cases when the ground state (GS) is formed by the states described by two spin quantum numbers $S$, at least one of them different from 0 . This approach is referred to as the degenerate frustration, since it implies nontrivial degeneracy of the GS multiplet.

Synthesis of the nanonuclear chromium rings and analysis of appropriate spin models induced the classification of frustration suggested in Ref. [3]. The Kahn degenerate frustration, present for specific "critical" values of $\alpha>0$, was assigned to the first type of frustration. The other two types distinguish domains of frustration with different total spin $S_{0}$ in the GS: the second type means $S_{0}<s$, whereas the third type of frustration was reserved to the case $S_{0}=s$, i.e. when this quantum number

*corresponding author; e-mail: antekm@amu.edu.pl is the same as in the nonfrustrated domain $\alpha \leq 0$. This classification was based on limited set of data and some refinements in this simplified schema has been proposed recently $[4,7]$.

The LMT not only shows the upper limit $\mathcal{S}$ of the total spin number $S_{0}$ in the GS, i.e. $S_{0} \leq \mathcal{S}$, but for a wide class of bipartite systems the so-called Lieb-Mattis level order (LMLO) is observed [10, 12, 13]:

$$
E_{\min }(S+1)>E_{\min }(S) \text { for } S \geq \mathcal{S},
$$

where $E_{\min }(S)$ denotes the minimum energy in the band corresponding to a given total spin quantum number $S$. The systems considered in this paper are not bipartite, but performed calculations for many systems have shown that the LMLO is preserved even when the LMT assumptions are violated.

In this paper a survey of main results is given. The LMLO is described and analysed for the pentanuclear ring to confirm its validity and its importance in estimation of the defect magnitude $\alpha$.

\section{The ground states sequence}

A square of the total spin $S^{2}$ commutes with the Hamiltonian (1), so the total spin number $S$ can be used to label states. An additional label is provided by symmetry of the model considered: the system is invariant with respect to the reflection in the plane perpendicular to the defected bond. Therefore, states gain an extra in$\operatorname{dex} r= \pm 1$, which distinguishes the symmetric and the antisymmetric ones, respectively.

For $\alpha<0$ there is the unique GS with the total spin $S_{0}=s$ for all sizes $n$ and all spin numbers $s$. Simple algebraic calculations performed in the case $n=3$ show that this state is preserved in the domain $0 \leq \alpha<0.5=\alpha_{c}^{(1)}$, despite of the spin number $s>1 / 2$ [4]. For these values of the defect magnitude the third type of frustration is observed. At $\alpha_{c}^{(1)}=0.5$ the first point of the Kahn degenerate frustration is present $[4,11]$. Above this value the universal sequence of $2 s-1$ GS's with spin numbers 
$S_{0}<s$ (the second type of frustration) is observed with $2 s-2$ critical values $\alpha_{c}^{(j)}, 1<j<2 s$, at which degenerate frustration occurs. Above the last critical value $\alpha_{c}^{(2 s)}=s+1$ the third type of frustration is again observed, $S_{0}=s$. For increasing $\alpha>0$ the universal sequence of the total spin numbers $S_{0}$ is given as

$$
S_{0}=s, s-1, \ldots,\left\{\begin{array}{c}
2,1,0,1,2 \\
3 / 2,1 / 2,1 / 2,3 / 2
\end{array}\right\} \ldots, s-1, s,(3)
$$

where the upper (lower) row corresponds to the integer (half-integer) spin number $s>1 / 2$, respectively. This sequence starts with the symmetric GS $|s,+1\rangle$ and the in$\operatorname{dex} r$ alternates, so the final GS is labelled as $\left|s,(-1)^{2 s}\right\rangle$. For $s=1 / 2$ there is the unique critical value $\alpha_{c}^{(1)}=1$, at which the symmetric $|1 / 2,+1\rangle$ and the antisymmetric $|1 / 2,-1\rangle$ states have the same energy. Since the three-spin system is bipartite for any $\alpha$ [4], so this result, $S_{0} \leq s$, agrees with the LMT conclusions, but the sequence obtained provides not only the upper limit $\mathcal{S}=s$, but exact values of $S_{0}$ in a given domain $\alpha_{c}^{(j)}<\alpha<\alpha_{c}^{(j+1)}, 1 \leq j<2 s$ are determined.

Larger systems, with $n>3$, are no longer bipartite but in all investigated cases the same sequence (with different critical values $\alpha_{c}^{(j)}, 1 \leq j \leq 2 s$, of course) has been revealed [4]. For example, the sequences obtained for $n=5$ and $s=2,5 / 2$, and 3 are illustrated in Fig. 1 .

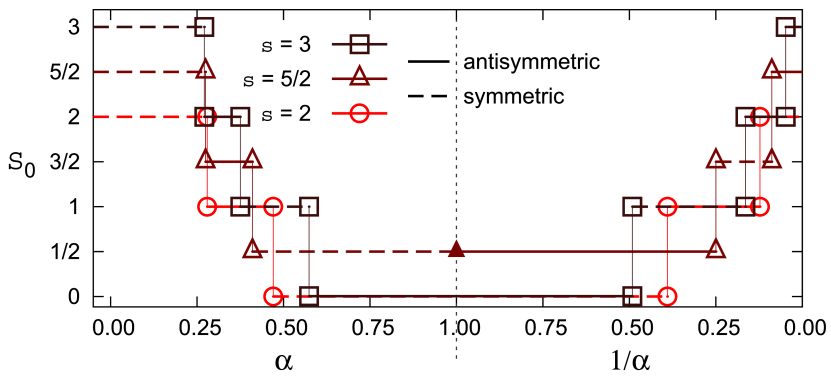

Fig. 1. The total spin $S_{0}$ in the GS as a function of $\alpha$ for $n=5$ and $s=2,5 / 2,3$. The symbols correspond to critical values of $\alpha$ the degenerate frustration is observed at. For the half-integer spin $s=5 / 2$ and $\alpha=1$ (denoted by a full triangle) the symmetry of the state with $S_{0}=$ $1 / 2$ is changed. Note that for $\alpha>1$ the values of $1 / \alpha$ are used.

Hence, these results strongly suggest that for any odd $n$ and any spin number $s$ there are $2 s+1$ different GS's separated by $2 s$ Kahn frustration points characterised by the enhanced degeneracy [4]. Thus the phenomenon of degenerate frustration is not so rare as anticipated earlier [3]. The coordinates of the corresponding level crossings $\alpha_{c}^{(j)}(1 \leq j \leq 2 s)$ for some sizes $n$ and spin numbers $s$ are exemplified in the supplemental material to Ref. [4].

For all half-integer spin numbers $S$ the level crossing of the GS's $|1 / 2, \pm 1\rangle$ is present at $\alpha_{c}^{(s+1 / 2)}=1$. This recovers the anticipated fourfold degeneracy [14], which is also present if the single-ion anisotropy is included [15]. For integers spin numbers $s$ in the interval $\alpha_{c}^{(s)}<\alpha<$ $\alpha_{c}^{(s+1)}$, which includes the value $\alpha=1$, the nonmagnetic GS with $S_{0}=0$ is observed, which has been determined earlier in some particular cases $[11,14]$.

\section{The Lieb-Mattis level order}

The LMT can be directly applied to the system with $n=3$, since it is bipartite for any $\alpha$ or the tetranuclear cluster [16]. Hence, the LMLO has to be present in this case. This result can be verified with some simple algebra (see also Fig. 2). It has occurred that the bipartiteness of a system is not a necessary condition, since the systems considered with $n>3$ are not bipartite (in a sense of the LMT), but the order of energies $E_{\min }(S)$ given by Eq. (2) is satisfied for all $S \geq S_{0}$. It means that the differences

$$
\Delta_{S}=E_{\min }(S)-E_{\min }(S-1) \geq 0
$$

for any $\alpha\left(\Delta_{S}=0\right.$ at the Kahn degenerate frustration points with $\left.\alpha=\alpha_{c}^{(j)}\right)$; as an example plots for $n=3,5$ and $s=2$ are presented in Fig. $2(1 \leq S \leq 2 n)$. Note that there are $s=2$ lines with zeros (the lowest ones) and the $2 s=4$ upper lines tends to linear functions with increasing slopes. The other lines (their number amounts to $(n-3) s$, so they are absent for $n=3)$ tends asymptotically to constant values. The LMLO, determined in Eq. (2), demands $\Delta_{S} \geq 0$, but the results obtained satisfy even stronger condition

$$
\Delta_{S+1}>\Delta_{S}, \quad S_{0}<S<n s,
$$

for an arbitrary fixed $\alpha$. This yields the field-driven transitions between GS's with different magnetisation $M \rightarrow M+1$ in an external magnetic field [4, 7]. Therefore, characteristic magnetisation steps should be observed and from their positions and widths the defect magnitude $\alpha$ can be directly estimated. For example, the cross-sections depicted in Fig. 2 for $\alpha=0.5,1.3$ give the magnetisation steps presented in Fig. 3, where the Zeeman term $-b M$, with $b=g \mu_{\mathrm{B}} B$, is added to the Hamiltonian (1). In both cases the zero-field $(b=0)$ GS has $S_{0}=0$ but even in a weak magnetic field these systems (the values of the parameter $\alpha$ ) can be easily distinguished.

\section{Conclusions}

The results obtained for many system sizes $n$ and spin numbers $s$ confirm the universal sequence of the total spin $S_{0}$ in the GS and presence of the LMLO. Note that the upper limit $S_{0} \leq s$ is obeyed despite the system size $n$. These facts can be easily determined from the LMT when $n=3$, but large rings are not bipartite, so some other properties of such systems should be considered. Note that in finite systems under question the third type of frustration, $S_{0}=s$, is always present in the domain $\left(0, \alpha_{c}^{(1)}\right)$ and the $2 s$ "critical" values of the parameter $\alpha$ determine systems with degenerate frustration. 


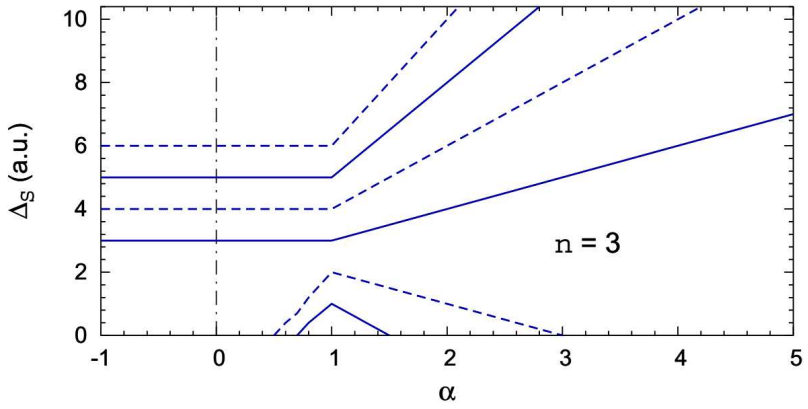

Fig. 2. The LMLO in a ring with a bond defect for $s=$ 2 and $n=3$ (top) or $n=5$ (bottom). The differences $\Delta_{S}$ are plotted for $S=1,2, \ldots, 2 n$ from bottom to top. Irrelevant segments (where $S \leq S_{0}$ ) are omitted. Zeros of $\Delta_{1}$ and $\Delta_{2}$ are placed at $\alpha=\alpha_{c}^{(j)}, j=1,2,3,4$. The dashed-dotted lines denote cross-sections used for plots of magnetisation steps.
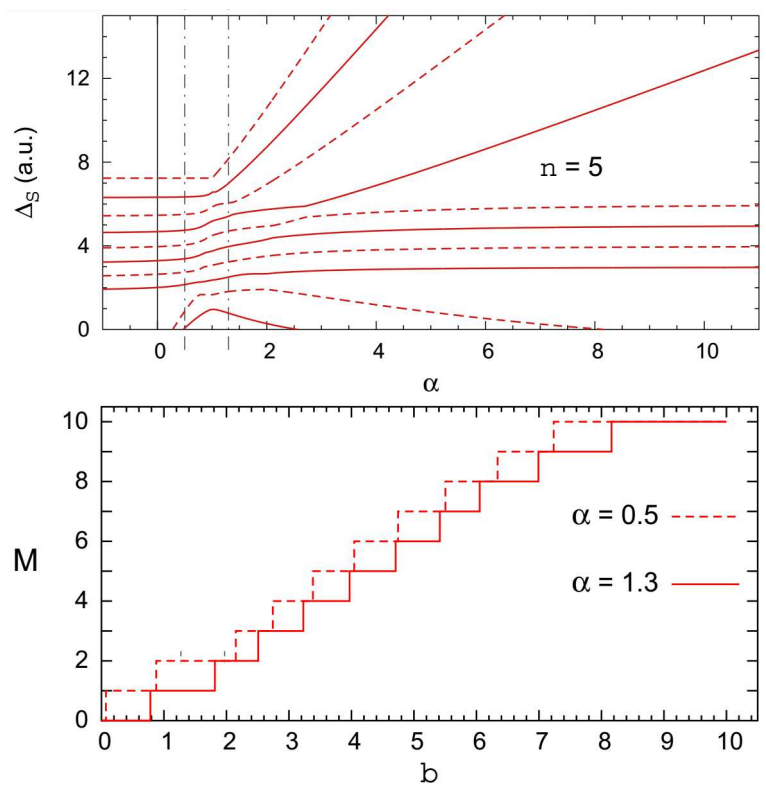

Fig. 3. Steps of the magnetisation $M$ in the ground state of the pentanuclear ring with a single bond defect $\alpha=0.5$ (dashed) and $\alpha=1.3$ (solid). The parameter $b=g \mu_{\mathrm{B}} B$ includes the Landé factor $g$, the Bohr magneton $\mu_{\mathrm{B}}$, and the external magnetic field $B$ along the $z$-th axis.

The model of ring-shaped nanomagnets reveals the LMLO despite the lack of bipartiteness. Moreover, the exact algebraic calculations (for $n=3$ ) and numerically exact results (for $n>3$ ) have indicated not only the LMLO $\left(\Delta_{S} \geq 0\right)$ but the increasing sequence of these differences. Hence, the magnetisation plateaus with the steps of the same height $\Delta M=1$ are predicted for fieldinduced transitions. Since properties of these steps depend on $\alpha$, then with a large enough external magnetic field one may distinguish systems with the same total spin in the ground state, but with different value of the parameters $\alpha$. These steps suggest also presence of the magnetocaloric effect, like in the centred hexagon discussed in Ref. [1]. However, in the systems discussed here wide plateaus are present in a rather nonphysical domain of large defect magnitude $\alpha$.

\section{Acknowledgments}

We acknowledge access to the HPC resources in PSNC Poznań (Poland).

\section{References}

[1] J.W. Sharples, D. Collison, E.J.L. McInnes, J. Schnack, E. Palacios, M. Evangelisti, Nature Commun. 5, 5321 (2014).

[2] M.L. Baker, T. Guidi, S. Carretta, J. Ollivier, H. Mutka, H.U. Güdel, G.A. Timco, E.J.L. McInnes, G. Amoretti, R.E.P. Winpenny, P. Santini, Nature Phys. 8, 906 (2012).

[3] M.L. Baker, G.A. Timco, S. Piligkos, J.S. Mathiesone, H. Mutka, F. Tuna, P. Kozłowski, M. Antkowiak, T. Guidi, T. Gupta, H. Rath, R.J. Woolfson, G. Kamieniarz, R.G. Pritchard, H. Weihe, L. Cronin, G. Rajaraman, D. Collison, E.J.L. McInnes, R.E.P. Winpenny, Proc. Natl. Acad. Sci. USA 109, 19113 (2012).

[4] G. Kamieniarz, W. Florek, M. Antkowiak, Phys. Rev. B 92, 140411(R) (2015).

[5] M.L. Baker, T. Lancaster, A. Chiesa, G. Amoretti, P.J. Baker, C. Barker, S.J. Blundell, S. Carretta, D. Collison, H.U. Güdel, T. Guidi, E.J.L. McInnes, J.S. Möller, H. Mutka, J. Ollivier, F.L. Pratt, P. Santini, F. Tuna, P.L.W. Tregenna-Piggott, I.J. VitoricaYrezabal, G.A. Timco, R.E.P. Winpenny, Chem. Europ. J. 22, 1779 (2016).

[6] O. Cador, D. Gatteschi, R. Sessoli, A.-L. Barra, G. Timco, R.E.P. Winpenny, J. Magn. Magn. Mater. 290-291, 55 (2005).

[7] M. Antkowiak, P. Kozłowski, G. Kamieniarz, G.A. Timco, F. Tuna, R.E.P. Winpenny, Phys. Rev. B 87, 184430 (2013).

[8] J. Schnack, Dalton Trans. 39, 4677 (2010).

[9] P. Kozłowski, M. Antkowiak, G. Kamieniarz, J. Nanopart. Res. 13, 6093 (2011).

[10] E. Lieb, D. Mattis, J. Math. Phys. 3, 749 (1962).

[11] O. Kahn, Chem. Phys. Lett. 265, 109 (1997).

[12] J. Richter, N. Ivanov, K. Retzlaff, A. Voigt, J. Magn. Magn. Mater. 140-144, 1611 (1995).

[13] J. Schnack, M. Luban, Phys. Rev. B 63, 014418 (2000).

[14] K. Bärwinkel, P. Hage, H.-J. Schmidt, J. Schnack, Phys. Rev. B 68, 054422 (2003).

[15] G. Kamieniarz, P. Kozłowski, M. Antkowiak, P. Sobczak, T. Ślusarski, D.M. Tomecka, A. Barasiński, B. Brzostowski, A. Drzewiński, A. Bieńko, J. Mroziński, Acta Phys. Pol. A 121, 992 (2012).

[16] M. Sobocińska, M. Antkowiak, M. Wojciechowski, G. Kamieniarz, J. Utko, T. Lis, Dalton Trans. 45 7303 (2016) 